\title{
Synthesis and antimicrobial activity of Bis-Derivatives of $3 \mathrm{a}^{\prime}$, 6a'Dihydro-2'H-Spiro[Indole-3,1'-Pyrrolo[3,4-c]Pyrrole]-2,4',6'(1H, 3'H, 5'H)-Trione
}

\author{
Ruslan Gr. Redkin", Evgeniya I. Syumka, Leonid A. Shemchuk, Valentin P. Chernykh \\ ${ }^{1}$ National University of Pharmacy, Pushkinska Str. 53, Kharkiv, 61001, Ukraine.
}

\begin{tabular}{l} 
ARTICLE INFO \\
\hline Article history: \\
Received on: 19/02/2017 \\
Accepted on: 06/04/2017 \\
Available online: $30 / 06 / 2017$ \\
\hline Key words: \\
Isatins, $\alpha$-amino acids, 2- \\
oxindoles; bis-spirocyclic \\
systems; bis-maleimides, $1,3-$ \\
cycloaddition, antimicrobial \\
activity.
\end{tabular}

\section{INTRODUCTION}

Isatin (1) and its derivatives are highly effective initial substrates for the synthesis of a wide range of heterocyclic compounds, particularly derivatives of 2-spiro-oxindole (Joaquim et al., 2001; Pandeya et al., 2005). The analysis of international experience shows the great attention of researchers to isatin as an initial synthon for the synthesis of spiroheterocyclic platform (Pavlovska et al., 2016; Singh and Desta, 2012), which has been named privileged structure for the synthesis and search for new biologically active compounds, in particular, novel antimicrobial agents (Ball-Jones et al., 2012). Thus, compounds with antibacterial action (Pandeya et al., 1999; Rane et al., 2016) and antiviral activity against smallpox virus

\footnotetext{
* Corresponding Author

Dr. Ruslan Gr. Redkin, National University of Pharmacy, Pushkinska Str. 53, Kharkiv, 61001, Ukraine.Email: ruslan.red.chem @ gmail.com
}

(Pirrung et al., 2005) were discovered among derivatives of isatin; bis-Schiff base-based isatin exhibit a wide spectrum of antibacterial, antiviral and antifungal activity (Aliasghar et al., 2007). Some isatin $\beta$-phenylhydrazones approached to the level of antimicrobial activity against Mycobacterium tuberculosis H37Rv streptomycin and ciprofloxacin in vitro (Karki et al., 2011). Some novel isatin derivatives of sultams have been synthesized and these compounds possess significant antibacterial and moderate antifungal activities (Harikrishna and Ravindranath, 2015). In order to enhance the antibacterial action of fluoroquinolones, Mannich bases were synthesized on the basis of isatin, ciprofloxacin and lomefloxacin, respectively (Pandeya et al., 1998).

Derivatives of spiro[indole-3,2'-pyrrolo[3,4-c]pyrrole] (3) have bioisosteric properties with regard to their natural prototypes to the spiro[pyrrolidine-3,3'-oxindole] (2) core in several alkaloids (5-6) and their spiro structure is not flat, which provides for a much greater affinity to biotargets (Syumka et al., 2015). 
That is why the synthetic methods lead to these compounds, their chemical and pharmacological properties are of great interest in the last few decades (Yu et al., 2015). In addition, some 3,3'-pyrrolidinyl-dispirooxindole compounds have also been demonstrated to show promising bioactivities, including antimicrobial (7, 10) (Babu et al., 2008) and anticancer (8) (Arun et al., 2013) properties. Due to their unique three-dimensional structural features, spirooxindoles have been identified as privileged chemotypes for antiviral drug development, e.g. (9) inhibitor activity against HIV (Ye et al., 2016) (Figure 1). At the same time, antimicrobial potential synthetic dispirooxindolic compounds have not been investigated.

In particular, the key idea for drug-design of new chemotherapeutic agents based on synthetic spirocyclic platform is the possibility of introducing further aliphatic substituents located in the areas of the ATP-binding pocket above or below the plane of the interaction with the hinge elements which are present in certain microbial cell kinases (Osolodkin et al., 2013). Because the vast majority of inhibitors of microbial enzymes known to date comprise planar or flattened structures, they cannot bind with specific biological target cavities above or below the plane of the molecule, which does not contribute to the selectivity of the inhibition.

Considering all the above-stated facts, the search for new antimicrobial agents among derivatives of spiro[2-oxindole-3,2'pyrrole] $(\mathbf{3}, \mathbf{8 - 1 0})$ is a promising direction for the development of new drugs having antimicrobial action.

The aim of this work is as follows: synthesis and search for drug-like molecules and estimation of their antimicrobial potential among hexamethylene-N,N'-bis-derivatives of spiro[indole-3,2'-pyrrolo[3,4-c]pyrrole] synthesized for the first time. The study compounds were obtained by multi-component one-pot condensation of isatins, $\alpha$-amino acids and 1,6bismaleimidohexane.

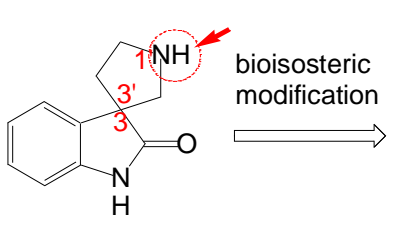

2

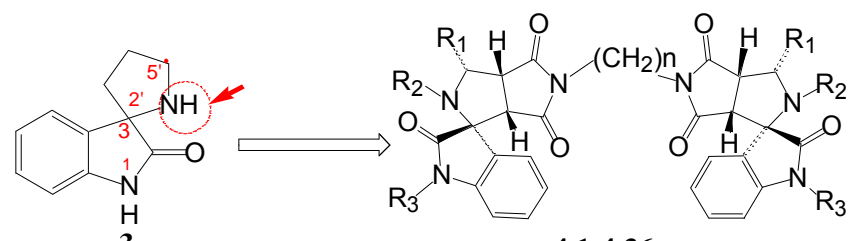

4.1-4.36

Spiro[pyrrolidine-3,3'-oxindole]

Spiro[pyrrolidine-3,2'-oxindole]

Bis-spiro[pyrrolidine-3,2'-oxindole] (target compounds)

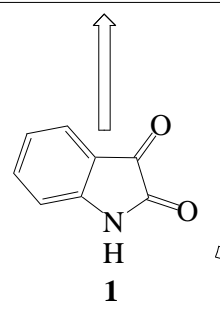

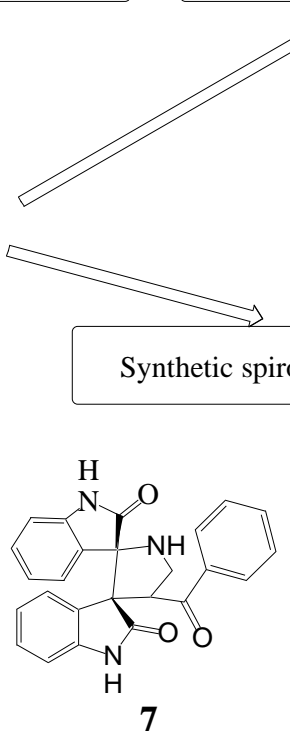

antimicrobial activity

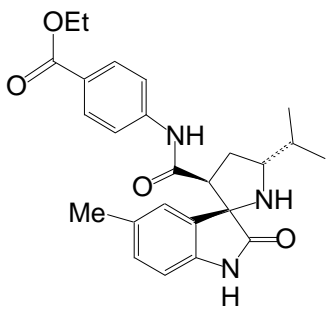

9

inhibitory activity against HIV

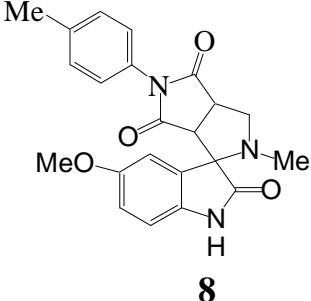

anti-tumor activity

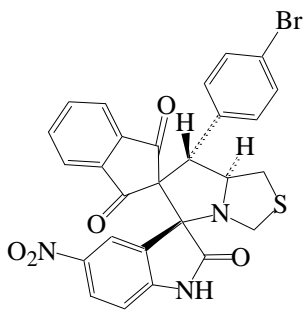

10

antitubercular activity

Fig. 1: Background for the synthesis of the target compounds 


\section{MATERIALS AND METHODS}

Starting isatins and bis-hexamethylenemaleimide were obtained from commercial sources and used without further purification. Melting points were obtained on a Gallenkamp melting point apparatus, Model MFB-595 in open capillary tubes. ${ }^{1} \mathrm{H}$ NMR and ${ }^{13} \mathrm{C}$ NMR spectras were recorded on a Varian WXR $(200 \mathrm{MHz})$ spectrometer in DMSO- $d_{6}$ using TMS as an internal standard (chemical shifts in parts per million); the references at the signal of the solvent $39.5 \mathrm{ppm}$ for DMSO- $d_{6}$. Elemental analyses were carried out using Carlo Erba CHNS-O EA 1108 analyzer. Mass spectra were taken usang a Varian 1200L DIP (EI, $70 \mathrm{eV}$ ).

The progress of reactions and purity of the obtained compounds were monitored by TLC on Silufol UV-254 plates in the systems acetone-heptane, 4:1, $\mathrm{H}_{2} \mathrm{O}-\mathrm{MeOH}, 1: 9$. Commercially available reagents and solvents were used without additional purification.

Method for preparation of $1^{\prime}-\mathrm{R}_{3}-2^{\prime}-\mathrm{R}_{2}-3^{\prime}-\mathrm{R}_{1}-5^{\prime}-\left(6-\left\{1^{\prime}-\mathrm{R}_{3}-\right.\right.$ 2'-R $\mathrm{R}_{2}-3^{\prime}-\mathrm{R}_{1}-2,4^{\prime}, 6^{\prime}$-trioxo-1,2,3',3'a,4',5',6',6'a-octahydro -2'H-spiro [indole-3,1'-pyrrolo[3,4-c]pyrrole]-5'-yl\}hexyl)- 1,2,3',3'a,4',5',6', 6'a-octahydro-2'H-spiro[indole-3,1'-pyrrolo[3,4-c]pyrrole] -2,4',6'triones (4.1-4.36)

A mixture of the corresponding isatins 1 ( $2 \mathrm{mmol}), \alpha-$ amino acid $2(2 \mathrm{mmol})$, and 1,6-bismaleimidohexane $(1 \mathrm{mmol})$ in a mixture of $i-\mathrm{PrOH}-\mathrm{H}_{2} \mathrm{O}(3: 1,4 \mathrm{ml})$ was refluxed for 3-8 $\mathrm{h}$, the reaction progress was checked by TLC and by changes in the reaction mixture color from red to yellow. The solution was cooled and placed into a freezer at $-5^{\circ} \mathrm{C}$ for $24 \mathrm{~h}$ for compounds 4.1-4.36, or obtained precipitate was filtered off, washed with $i-\mathrm{PrOH}$, and recrystallized, or the reaction mixture was then poured onto ice, and resulting precipitate was filtered and recrystallized from mixture of DMF-EtOH (1:1).

\section{Antimicrobial activity study}

Microbiological experiment was performed by the Microbiology Department of Donetsk National Medical University (Ukraine). According to the WHO recommendations (Balouiri et al., 2016; Patel et al., 2015), the following strains of microorganisms were used to estimate the activity of the tested compounds: Escherichia coli ATCC 25922, Enterobacter cloacae №487, Klebsiella pneumoniae No. 247, Pseudomonas aeruginosa ATCC 27853, Staphylococcus aureus ATCC 25923, Staphylococcus epidermidis №235, Candida albicans ATCC653/885. Inoculum's density was 107 cells per $1 \mathrm{~mL}$ of media and was determined by comparing with McFarland standard. 18 to 24-hour old culture of the microorganism was used for the test. The studied compounds were introduced as $0.3 \mathrm{ml}$ DMSO $(100 \mu \mathrm{g} / \mathrm{mL})$ solution aliquots. To prepare serial dilutions of substances, solution of basic solids synthesized in a solvent dimethylsulfoxide (DMSO) were prepared. Then we prepared a series of two-fold dilution working solution in sterile distilled water at concentrations 10 times higher than the maximum concentration required for this study. The $\mathrm{pH}$ value of the initial solution was about 6.5 units. The inhibiting effect (Minimum
Inhibitory concentration (MIC) value) of the substances on microorganisms was determined using the method of serial dilutions in dense nutrient medium - meat-peptone agar (MPA). The MIC values were recorded after incubation at $28 \pm 2^{\circ} \mathrm{C}$ for $72 \mathrm{hrs}$. Solutions were aseptically brought to bottles with autoclave-sterilized and cooled to $50^{\circ} \mathrm{C}$ agar (1 part of the substance and 9 parts of the agar), and poured in Petri dishes with a layer thickness of 3-4 $\mathrm{mm}$. The inoculum suspension was prepared using the Densi-La-Meter apparatus (made by PLIVALachema, Czech Republic; 540-nm wavelength). The cultures were synchronized using the low temperature conditions $\left(4{ }^{\circ} \mathrm{C}\right)$. When the agar hardened, a drop of prepared suspensions of different strains of microorganisms at a concentration $107 \mathrm{CFU} / \mathrm{ml}$ was placed onto the surface of agar using a bacteriological loop (with the loop diameter of $3 \mathrm{~mm}$ ). The corresponding concentration was prepared by 10 -times dilution of the initial suspension of microorganisms equalling to 0.5 units by the McFarland turbidity scale $(108 \mathrm{CFU} / \mathrm{mL})$. Mueller-Hinton agar was employed (HIMedia Laboratorles Pvt. Ltd India) for bacteria. The strain of Candida albicans was cultivated using Sabouraud agar (HIMedia Laboratorles Pvt. Ltd India).

All molecular descriptors were calculated using the Molinspiration Cheminformatics v2016.09 software system, 2016 (Molinspiration property engine v2016.10, Bratislava University, Slovakia) available online at http://www.molinspiration.com. The authors used a standard IBM PC-compatible personal workstation (PIV CPU clocked at 1.4 $\mathrm{GHz}, 512 \mathrm{MB} \mathrm{RAM}$ ) running under Windows 2000 and Microsoft Office 2003.

\section{RESULTS AND DISCUSSION}

For this purpose, we synthesized bis-derivatives of 1,6hexyliden 3a',6a'-2'H-dihydro-spiro[indole-3,1'-pyrrolo[3,4c] pyrrole]-2,4',6' $\left(1 H, 3^{\prime} H, 5^{\prime} H\right)$-trione and studied antibacterial properties of 36 synthesized compounds vs. standard test strains and determined their antimicrobial potential.

Three-component condensations of isatin with amino acids and dipolarophiles containing an electron withdrawing substituents activated $\pi$-bond is the most promising method for forming the spiro heterocyclic system of [indole-3,2'-pyrrolo[3,4c]pyrrole] (Pavlovskaya et al. 2013). Therefore, this approach has been used by us for the synthesis of target symmetric bis-spiro oxindole derivatives (4.1-4.36). The target 4.1-4.36 bis-derivatives were obtained using a one-pot procedure and three-component domino cyclocondensation of isatins $\mathbf{1}\left(\mathrm{R}^{3}=\mathrm{H}, \mathrm{CH}_{3}, \mathrm{All}, \mathrm{Bn}\right)$ between $\alpha$-amino acids 11 (sarcosine, glycine, $L$-proline, $L$-serine, $L$-valine, $\quad L$-isoleucine, $L$-phenylglicine, $L$-phenylalanine, $\quad L$ thyrosine, $L$-methyonine, $L$-glutaminic acid and $L$-glutamine) and 1,6-bismaleimidohexane 12. The mechanism of interaction is the formation of unstable azomethine-ylides in situ as a result of decarboxylation of $\alpha$-amino acids and isatin products, with subsequent 1,3-dipolar cycloaddition of the olefinic bond by symmetrical dipolarophile $\mathbf{1 2}$. 
2<smiles>[R3]N1C(=O)C(=O)c2ccccc21</smiles>

1

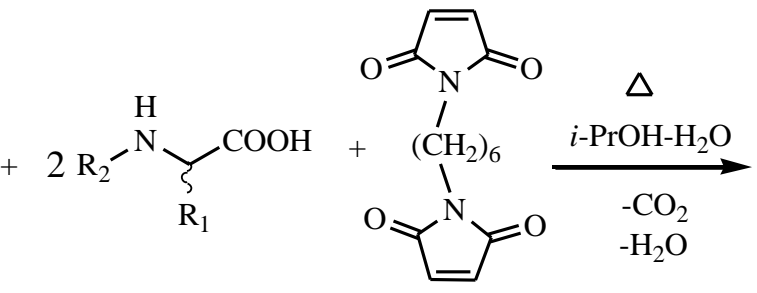

12

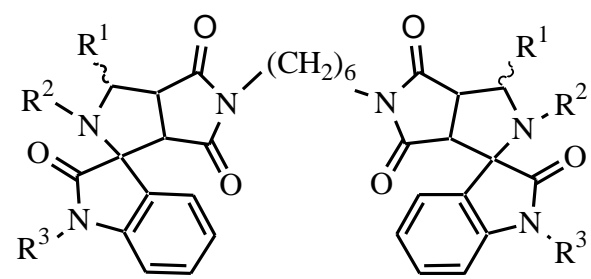

4.1-4.36

Fig. 2: Synthesis of 1'- $\mathrm{R}_{3}-2^{\prime}-\mathrm{R}_{2}-3^{\prime}-\mathrm{R}_{1}-5^{\prime}-\left(6-\left\{1^{\prime}-\mathrm{R}_{3}-2^{\prime}-\mathrm{R}_{2}-3^{\prime}-\mathrm{R}_{1}-2,4^{\prime}, 6^{\prime}-\right.\right.$ trioxo-1,2,3',3'a,4',5',6',6'a-octahydro-2'H-spiro[indole-3,1'-pyrrolo[3,4-c]pyrrole]-5'yl \}hexyl)-1,2,3',3'a,4',5',6',6'a-octahydro-2'H-spiro[indole-3,1'-pyrrolo[3,4-c]pyrrole]-2,4',6'-triones (4.1-4.36)

Table 1: Optimization of solvent effect on the model reaction ${ }^{\mathrm{a}}$.

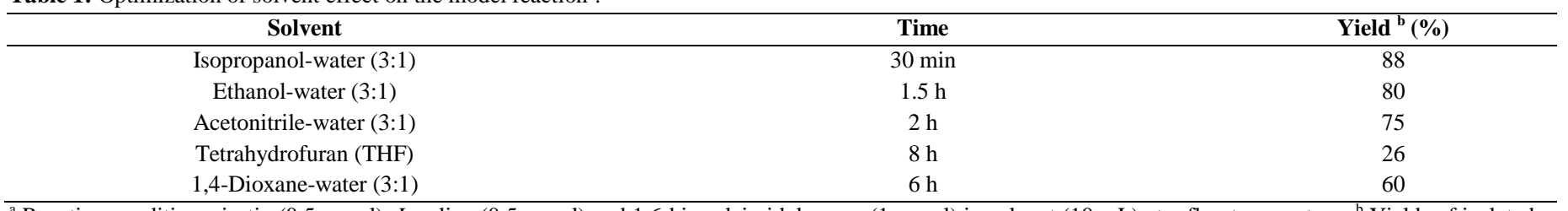

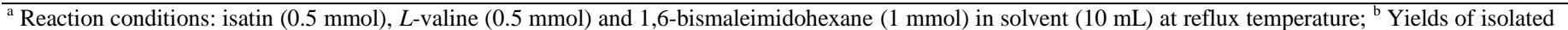
product 4.11 .

It was found that the regioselectivity of this reaction depends on the ratio of reagents. So, symmetrical bis-derivatives 4.1-4.36 can be obtained only at a molar double excess $\mathbf{1}$ and $\mathbf{1 1}$ in moderate to excellent yields (Figure 2, Table 1).

The reaction time largely depend on the reactivity of the employed $\alpha$-amino acids. The longest reaction time (5-7 h) was found for sarcosine and glycine $(5 \mathrm{~h})$, while the fastest reaction (30-45 min) was found for $L$-valine, $L$-isoleucine and $L$-proline as substrates. Selecting an appropriate solvent is of critical importance for effective synthesis. To optimize the reaction solvent, the reaction of isatin, $L$-valine, and 1,6bismaleimidohexane was carried out in different organic solvents such as mixture (1:3) of isopropanol, ethanol, acetonitrile, 1,4dioxane with water and THF at reflux condition. Water is desired to better dissolving of the $L$-amino acids in the reaction mixture. The results are summarized in Table 1 . It was shown that the reactions in refluxing isopropanol-water (3:1) delivered the best results. This method has the advantages of mild reaction conditions, high atom economy and excellent yields.

In our case, spirooxindols 4.1-4.36 are exclusively formed. All new cycloadducts obtained by the above method were characterized by mass spectrometry, ${ }^{1} \mathrm{H}$ and ${ }^{13} \mathrm{C}$ NMR for compounds 4.1, 4.3, 4.19, 4.34 (Table 2).

Signals for all proton-containing fragments are present in ${ }^{1} \mathrm{H}$ NMR spectras of compounds 4.1-4.36 (Table 2). Assignment of the signals of $\mathrm{NH}$ and $\mathrm{OH}$ groups were made with the aid of deuterium exchange with $\mathrm{D}_{2} \mathrm{O}$. The $\mathrm{NH}$-proton of the oxindole moiety appeared as a singlet between $10.38-10.86 \mathrm{ppm}$. The resonance of the methine protons of the pyrrolo[3,4-c]pyrrole system was displayed as a doublet at 3.40-3.50 ppm for the H-6a' proton, a triplet at 3.50-3.60 ppm for the $\mathrm{H}-3 \mathrm{a}^{\prime}$ proton, and a multiplet for the H-3' proton, which was located at 4.00-4.40 ppm in the spectra of compounds 4.1-4.36. Values of the coupling constants of the H-3a' and H-6a' protons and of H-3' and H-3a' amounted to 7-8 $\mathrm{Hz}$, which may point at their cis orientation. The benzylic ( $3^{\prime}-\underline{\mathrm{C}}_{2} \mathrm{Ar}$ ) protons in compounds $4.5,4.19,4.20$ were detected as multiplets with $\mathrm{H}-3$ ' protons at $\delta 4.10-4.25 \mathrm{ppm}$ and the benzylic ( $\left.\mathrm{N}-\underline{\mathrm{C}}_{2} \mathrm{Ar}\right)$ 4.25-4.31 are exhibited a singlet at $\delta$ 4.65-5 $\mathrm{ppm}$. The sharp singlet at $\delta 3.01-3.10$ due to the N-methyl protons was seen for componds $\mathbf{4 . 3 2}-\mathbf{4 . 3 6}$. The signals of aliphatic protons of hexamethylene residue, which are observed as signal of $\mathrm{CH}_{2} \mathrm{CH}_{2}$ groups two multiplets at $\delta 0.83-1.29$ and $\delta 1.11-1.75 \mathrm{ppm}$ and the signal of $5^{\prime}-\mathrm{NCH}_{2}$ two fragments is present in the region from $\delta 2.53$ up to $3.52 \mathrm{ppm}$. The main feature of the ${ }^{13} \mathrm{C}$ spectra of compounds 4.1-4.36 is the presence of the signal of the $3 \mathrm{C}$-spiro nucleus. For example, in the ${ }^{13} \mathrm{C}-\mathrm{NMR}$ spectrums of compounds 4.1, 4.3, 4.19, 4.34, the signal at $\delta 67-68 \mathrm{ppm}$ due to the spirocarbons was observed.

Table 2: The properties of $1^{\prime}-R_{3}-2^{\prime}-R_{2}-3^{\prime}-R_{I}-5^{\prime}-\left(6-\left\{1^{\prime}-R_{3}-2^{\prime}-R_{2}-3^{\prime}-R_{I}-2,4^{\prime}, 6^{\prime}-\right.\right.$ trioxo-1,2,3',3'a, 4',5',6',6'a-octahydro-2' $H$ - spiro[indole-3,1'- pyrrolo[3,4c]pyrrole] -5'-yl \}hexyl)-1,2,3',3'a,4',5',6',6'a-octahydro-2' $H$-spiro[indole-3,1'pyrrolo[3,4-c]pyrrole]-2,4',6'-triones (4.1-4.36).

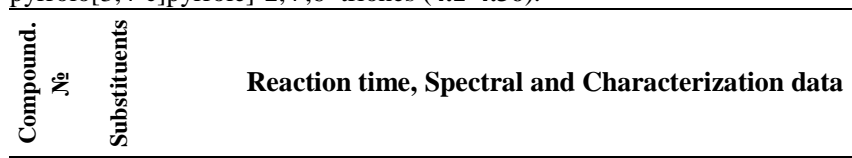

Compound 4.1.: time $1 \mathrm{~h} ; 75, \%$ yield; $190{ }^{\circ} \mathrm{C} * ;{ }^{1} \mathrm{H}$ NMR $(200$ $\mathrm{MHz}$, DMSO- $\left.d_{6}\right) \delta 1.29\left(\mathrm{~m}, 4 \mathrm{H}, \mathrm{CH}_{2} \mathrm{CH}_{2}\right), 1.43(\mathrm{~m}, 4 \mathrm{H}$, $\mathrm{CH}_{2} \mathrm{CH}_{2}$ ), 3.59-3.86 (m, 4H, $2 \times 6$ 'a-C태, $2 \times 5^{\prime}-\mathrm{NC}_{2}$ ), 4.07-4.20

$\begin{array}{ll}\text { II } & \left(2 \mathrm{H}, 2 \times 3^{\prime} \mathrm{a}-\mathrm{CH}, J=7.01 \mathrm{~Hz}\right), 4.34\left(\mathrm{~d}, 2 \mathrm{H}, 2 \times 2^{\prime}-\mathrm{NH}, J=4.27 \mathrm{~Hz}\right), \\ & 5.40-5.52\left(\mathrm{~m}, 2 \mathrm{H}, 3^{\prime}-\mathrm{CH}\right), 6.98(\mathrm{~s}, 5 \mathrm{H}, \mathrm{ArH}), 7.21(\mathrm{~m}, 9 \mathrm{H}, \mathrm{ArH}),\end{array}$ 대 $7.34(\mathrm{~m}, 4 \mathrm{H}, \mathrm{ArH}), 10.38(\mathrm{~s}, 2 \mathrm{H}, 1-\mathrm{NH}) \mathrm{ppm} ;{ }^{13} \mathrm{C}-\mathrm{NMR}(75$ $\dot{\rightarrow} \cong \mathrm{MHz}$, DMSO-d $\left.d_{6}\right) \delta 25.30,25.60,25.74,27.12,27.76,37.67$, 数 $38.68,38.89,39.09,39.51,39.72,39.93,49.34,50.59,59.45$, $\approx \quad 61.84,67.11,109.08,120.79,126.42,126.91,127.14,127.33$, $127.40,128.05,128.83,134.27,139.29,142.13,174.46,174.98$, 180.67 ppm; EI-MS for $\mathrm{C}_{44} \mathrm{H}_{40} \mathrm{~N}_{6} \mathrm{O}_{6}(\mathrm{M}+1) 748.8$. 


\section{Compound 4.2.: time $40 \mathrm{~min} ; 81$, \% yield; $150-152{ }^{\circ} \mathrm{C} ;{ }^{1} \mathrm{H}$ NMR (200 MHz, DMSO- $\left.d_{6}\right) \delta 1.29\left(\mathrm{~m}, 4 \mathrm{H}, \mathrm{C}_{2} \mathrm{C}_{2}\right), 1.49(\mathrm{~m}, 4 \mathrm{H}$, $\left.\mathrm{C}_{2}{ }_{2} \underline{\mathrm{H}}_{2}\right), 2.27$ (m, 4H, $\left.2 \times 5^{\prime}-\mathrm{NCH}_{2}\right), 3.26-3.41(\mathrm{~m}, 14 \mathrm{H}, 2 \times 6$ 'a- $\left.\mathrm{CH}, 2 \times \underline{\mathrm{H}}_{2} \mathrm{C}_{2}{ }_{2} \underline{\mathrm{H}}_{2}\right), 3.43-3.60$ (m, $\left.4 \mathrm{H}, 2 \times 3{ }^{\prime} \mathrm{a}-\mathrm{CH}\right), 4.20$ (q, $2 \mathrm{H}$, $\left.2 \times 3^{\prime}-\mathrm{CH}, J=7.02 \mathrm{~Hz}\right), 6.73-6.96(\mathrm{~m}, 6 \mathrm{H}, \mathrm{ArH}), 7.11-7.31(\mathrm{~m}$, $2 \mathrm{H}, \mathrm{ArH}), 10.52$ (s, $2 \mathrm{H}, 2 \times 1-\mathrm{NH}) \mathrm{ppm}$; EI-MS for $\mathrm{C}_{38} \mathrm{H}_{40} \mathrm{~N}_{6} \mathrm{O}_{6}$ $(\mathrm{M}+1) 676.7$. \\ Compound 4.3.: time $2 \mathrm{~h}$; 62 , \% yield; $260-262{ }^{\circ} \mathrm{C} ;{ }^{1} \mathrm{H}$ NMR $\left(200 \mathrm{MHz}, \mathrm{DMSO}-d_{6}\right) \delta 1.13\left(\mathrm{~d}, 6 \mathrm{H}, 2 \times 3^{\prime}-\mathrm{C}_{3}, J=6.41 \mathrm{~Hz}\right), 1.32$ $\left(\mathrm{m}, 4 \mathrm{H}, \mathrm{CH}_{2} \mathrm{CH}_{2}\right), 1.52\left(\mathrm{~m}, 4 \mathrm{H}, \mathrm{CH}_{2} \mathrm{CH}_{2}\right), 3.17-3.24(\mathrm{~d}, 2 \mathrm{H}$, III $2 \times 6$ 'a-CH, $J=7.9 \mathrm{~Hz}), 3.29-3.47\left(\mathrm{~m}, 6 \mathrm{H}, 2 \times 3\right.$ 'a- $\left.\mathrm{CH}, 2 \times 5^{\prime}-\mathrm{NCH}_{2}\right)$, 业 $\quad 3.65\left(\mathrm{~d}, 2 \mathrm{H}, 2 \times 2^{\prime}-\mathrm{NH}, J=4.88 \mathrm{~Hz}\right), 4.19-4.38\left(\mathrm{~m}, 2 \mathrm{H}, 2 \times 3^{\prime}-\mathrm{CH}\right)$, $\approx \quad 6.67-6.95(\mathrm{~m}, 6 \mathrm{H}, \mathrm{ArH}), 7.08-7.27(\mathrm{~m}, 2 \mathrm{H}, \mathrm{ArH}), 10.33(\mathrm{~s}, 2 \mathrm{H}$, $2 \times 1-\mathrm{NH}) \mathrm{ppm} ;{ }^{13} \mathrm{C}-\mathrm{NMR}\left(75 \mathrm{MHz}, \mathrm{DMSO}-d_{6}\right) \delta 16.90,25.72$, 歪 $25.77,27.16,37.95,38.87,39.07,39.91,40.12,48.56,51.74$, $\simeq \quad 51.98,67.79,109.15,120.92,126.29,127.51,128.94,142.12$, 175.00, 176.33, $180.42 \mathrm{ppm}$; EI-MS for $\mathrm{C}_{34} \mathrm{H}_{36} \mathrm{~N}_{6} \mathrm{O}_{6}(\mathrm{M}+1)$ 624.7 .}

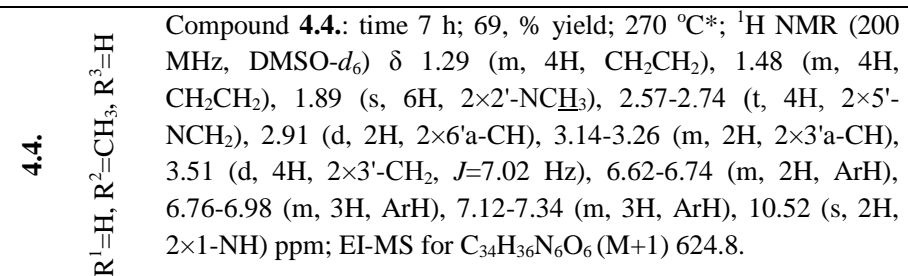

Compound 4.5.: time $1 \mathrm{~h} ; 55, \%$ yield; $275-278{ }^{\circ} \mathrm{C} ;{ }^{1} \mathrm{H}$ NMR ठํ. $\left(200 \mathrm{MHz}, \mathrm{DMSO}-d_{6}\right) \delta 1.37\left(\mathrm{~m}, 4 \mathrm{H}, \mathrm{CH}_{2} \mathrm{CH}_{2}\right), 1.57(\mathrm{~m}, 4 \mathrm{H}$, II $\left.\mathrm{CH}_{2} \mathrm{CH}_{2}\right), 2.97-3.29\left(\mathrm{~m}, 8 \mathrm{H}, 2 \times 3\right.$ 'a-CH, $2 \times 6$ 'a-CH, $2 \times 5$ '- $\left.-\mathrm{NCH}_{2}\right)$, i.

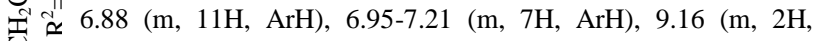
II ArOH), $10.30(\mathrm{~s}, 2 \mathrm{H}, 2 \times 1-\mathrm{NH})$ ppm; EI-MS for $\mathrm{C}_{46} \mathrm{H}_{44} \mathrm{~N}_{6} \mathrm{O}_{8}$ $\simeq \quad(\mathrm{M}+1)$ 808.9.

Compound 4.6.: time $5 \mathrm{~h}$; $80, \%$ yield; $182-185{ }^{\circ} \mathrm{C} ;{ }^{1} \mathrm{H}$ NMR (200 MHz, DMSO- $\left.d_{6}\right) \delta 1.16-1.34\left(\mathrm{~m}, 4 \mathrm{H}, \mathrm{CH}_{2} \mathrm{CH}_{2}\right), 1.39-1.60$ (m, $4 \mathrm{H}, \mathrm{CH}_{2} \mathrm{CH}_{2}$ ), $3.16\left(\mathrm{~m}, 4 \mathrm{H}, 2 \times 5^{\prime}-\mathrm{NCH}_{2}\right), 3.42$ (d, $2 \mathrm{H}, 2 \times 6$ ' a$\mathrm{CH}, J=7.9 \mathrm{~Hz}), 3.54\left(\mathrm{~m}, 4 \mathrm{H}, 2 \times 3^{\prime} \mathrm{a}-\mathrm{CH}\right), 3.75\left(\mathrm{t}, 2 \mathrm{H}, 2 \mathrm{H}, 2^{\prime}-\mathrm{NH}\right.$, $J=6.10 \mathrm{~Hz}), 4.24-4.43\left(\mathrm{t}, 3^{\prime}-\mathrm{CH}_{2} J=7.02 \mathrm{~Hz}\right), 6.71-6.93(\mathrm{~m}, 6 \mathrm{H}$, $\mathrm{ArH}), 7.18$ (t, 2H, ArH, J=7.63 Hz), 10.37 (s, 2H, 2×1-NH) ppm; EI-MS for $\mathrm{C}_{32} \mathrm{H}_{32} \mathrm{~N}_{6} \mathrm{O}_{6}(\mathrm{M}+1) 596.7$.

\footnotetext{
Compound 4.7.: time $1 \mathrm{~h} ; 74, \%$ yield; $180{ }^{\circ} \mathrm{C}^{*} ;{ }^{1} \mathrm{H}$ NMR (200 III MHz, DMSO-d $\left.d_{6}\right) \delta 1.23-1.39\left(\mathrm{~m}, 6 \mathrm{H}, \mathrm{CH}_{2} \mathrm{CH}_{2}, 2 \times \mathrm{CH}^{a} \mathrm{H}^{\mathrm{b}}-\mathrm{CH}_{2} \mathrm{~S}\right)$, 少 $1.45-1.67\left(6 \mathrm{H}, \mathrm{CH}_{2} \mathrm{CH}_{2}, 2 \times \mathrm{CH}^{a} \underline{\mathrm{H}}^{\mathrm{b}}-\mathrm{CH}_{2} \mathrm{~S}\right), 2.03\left(\mathrm{~s}, 6 \mathrm{H}, 2 \times \mathrm{SC}^{\mathrm{H}} 3\right)$,

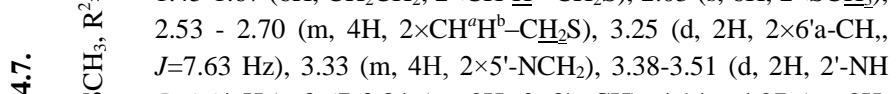
+ : $J=6.64 \mathrm{~Hz}), 3.67-3.84\left(\mathrm{~m}, 2 \mathrm{H}, 2 \times 3^{\prime} \mathrm{a}-\mathrm{CH}\right), 4.14-4.27(\mathrm{~m}, 2 \mathrm{H}$, $\left.2 \times 3^{\prime}-\mathrm{CH}\right), 6.69-6.91(\mathrm{~m}, 6 \mathrm{H}, \mathrm{ArH}), 7.17(\mathrm{t}, 2 \mathrm{H}, \mathrm{ArH}, J=8.09 \mathrm{~Hz})$, 10.35 (s, 2H, 2×1-NH) ppm; EI-MS for $\mathrm{C}_{38} \mathrm{H}_{44} \mathrm{~N}_{6} \mathrm{O}_{6} \mathrm{~S}_{2}(\mathrm{M}+1)$ 744.9.

Compound 4.8.: time $1 \mathrm{~h} ; 58, \%$ yield; $170-172{ }^{\circ} \mathrm{C} ;{ }^{1} \mathrm{H}$ NMR II $\left(200 \mathrm{MHz}, \mathrm{DMSO}-d_{6}\right) \delta 1.16\left(\mathrm{t}, 6 \mathrm{H}, 2 \times \mathrm{CH}_{3} \mathrm{CH}_{2} \mathrm{~S}, J=7.32 \mathrm{~Hz}\right)$, 光 $1.26-1.42\left(\mathrm{~m}, \quad 6 \mathrm{H}, \quad \mathrm{CH}_{2} \mathrm{CH}_{2}, 2 \times \mathrm{CH}^{a} \mathrm{H}^{\mathrm{b}}-\mathrm{CH}_{2} \mathrm{~S}\right), 1.53 \quad(6 \mathrm{H}$, $\left.\mathrm{CH}_{2} \mathrm{CH}_{2}, 2 \times \mathrm{CH}^{a} \underline{\mathrm{H}}^{\mathrm{b}}-\mathrm{CH}_{2} \mathrm{~S}\right), 1.84-2.12\left(\mathrm{~m}, 4 \mathrm{H}, 2 \times \mathrm{CH}^{a} \mathrm{H}^{\mathrm{b}}-\mathrm{CH}_{2} \mathrm{~S}\right)$, $2.62\left(\mathrm{~m}, 4 \mathrm{H}, 2 \times \mathrm{CH}_{3} \mathrm{CH}_{2} \mathrm{~S}\right), 3.24\left(\mathrm{~d}, 1 \mathrm{H}, 2^{\prime}-\mathrm{NH}, J=7.63 \mathrm{~Hz}\right), 3.40$ - 3.50 (t, $2 \mathrm{H}, 2 \times 3$ 'a-CH, ), 3.68-3.87 (m, 6H, $2 \times 6$ 'a-CH, $2 \times 5$ '-

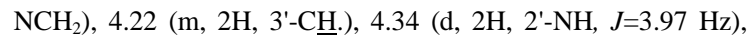
6.70-6.93 (m, 6H, ArH), 7.17 (t, 2H, ArH, J=7.32 Hz), 10.34 (s, $2 \mathrm{H}, 2 \times 1-\mathrm{NH}) \mathrm{ppm}$; EI-MS for $\mathrm{C}_{40} \mathrm{H}_{48} \mathrm{~N}_{6} \mathrm{O}_{6} \mathrm{~S}_{2}(\mathrm{M}+1) 772.9$.
}

\begin{tabular}{|c|c|c|}
\hline$\dot{\vec{\tau}}$ & 垔 & 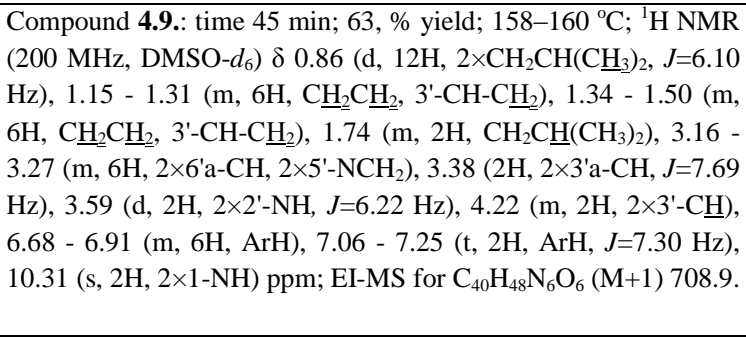 \\
\hline$\stackrel{\stackrel{\ominus}{\rightleftarrows}}{\dot{+}}$ & 垔 & 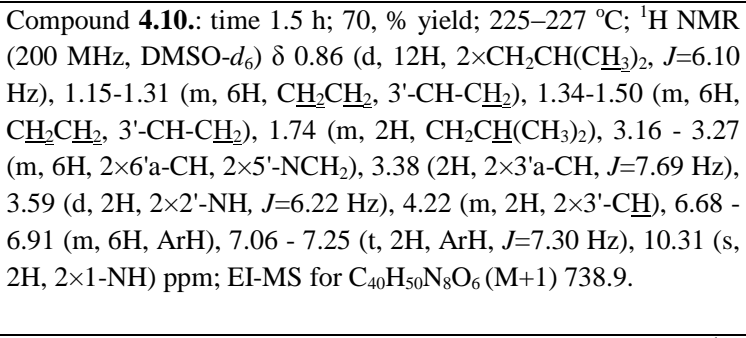 \\
\hline$\ddot{F}$ & 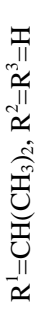 & $\begin{array}{l}\text { Compound 4.11.: time } 30 \mathrm{~min} ; 88, \% \text { yield; } 172-174{ }^{\circ} \mathrm{C} ;{ }^{1} \mathrm{H} \\
\mathrm{NMR}\left(200 \mathrm{MHz}, \mathrm{DMSO}-d_{6}\right) \delta 0.83\left(\mathrm{~d}, 12 \mathrm{H}, 2 \times \mathrm{CH}\left(\mathrm{C}_{3}\right)_{2},\right. \\
J=6.10 \mathrm{~Hz}), 1.11-1.20\left(\mathrm{~m}, 4 \mathrm{H}, \mathrm{CH}_{2} \mathrm{CH}_{2}\right), 1.21-1.38(\mathrm{~m}, 4 \mathrm{H}, \\
\left.\mathrm{CH}_{2} \mathrm{CH}_{2}\right), 1.38-1.59\left(\mathrm{~m}, 4 \mathrm{H}, 2 \times 5^{\prime}-\mathrm{NCH}_{2}\right), 3.23\left(\mathrm{~d}, 2 \mathrm{H}, 2 \times 6{ }^{\prime} \mathrm{a}-\right. \\
\mathrm{CH}, J=7.63 \mathrm{~Hz}), 3.48\left(\mathrm{t}, 2 \mathrm{H}, 2 \times 3{ }^{\prime} \mathrm{a}-\mathrm{CH} J=7.17 \mathrm{~Hz}\right), 3.64(\mathrm{~d}, 2 \mathrm{H} \text {, } \\
\left.2 \times 2^{\prime}-\mathrm{NH}, J=4.58 \mathrm{~Hz}\right), 3.68-3.84\left(\mathrm{~m}, 2 \mathrm{H}, 2 \times 3^{\prime}-\mathrm{CH}\right), 6.65-6.90 \\
(\mathrm{~m}, 6 \mathrm{H}, \mathrm{ArH}), 7.08-7.22(\mathrm{t}, 2 \mathrm{H}, \mathrm{ArH}, J=7.2 \mathrm{~Hz}), 10.29(\mathrm{~s}, 2 \mathrm{H} \text {, } \\
2 \times 1-\mathrm{NH}) \text { ppm; EI-MS for } \mathrm{C}_{38} \mathrm{H}_{44} \mathrm{~N}_{6} \mathrm{O}_{6}(\mathrm{M}+1) 680.8 .\end{array}$ \\
\hline$\underset{\overrightarrow{+}}{\dot{\sim}}$ & 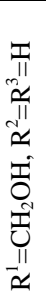 & $\begin{array}{l}\text { Compound 4.12.: time } 2 \mathrm{~h} ; 79, \% \text { yield; } 175-177{ }^{\circ} \mathrm{C} ;{ }^{1} \mathrm{H} \text { NMR } \\
\left(200 \mathrm{MHz}, \mathrm{DMSO}-d_{6}\right) \delta 1.15-1.31\left(\mathrm{~m}, 4 \mathrm{H}, \mathrm{C}_{2} \mathrm{C}_{2} \underline{\mathrm{H}}_{2}\right), 1.35- \\
1.50\left(\mathrm{~m}, 4 \mathrm{H}, \mathrm{C}_{2} \underline{\mathrm{H}}_{2}-\underline{\mathrm{H}}_{2}\right), 3.35-3.52\left(\mathrm{~m}, 6 \mathrm{H}, 2 \times 6{ }^{\prime} \mathrm{a}-\mathrm{CH}, 2 \times 5^{\prime}-\right. \\
\left.\mathrm{NCH}_{2}\right), 3.55-3.59\left(\mathrm{t}, 2 \mathrm{H}, 2 \times 3{ }^{\prime} \mathrm{a}-\mathrm{CH} J=7.2 \mathrm{~Hz}\right), 3.76(4 \mathrm{H}, \\
\left.2 \times \mathrm{C}_{2} \mathrm{O}\right), 3.86\left(\mathrm{~d}, 2 \mathrm{H}, 2 \times 2^{\prime}-\mathrm{NH}, J=6.22 \mathrm{~Hz}\right), 4.13-4.33(\mathrm{~m}, 2 \mathrm{H}, \\
\left.2 \times 3^{\prime}-\mathrm{CH}\right), 4.50\left(\mathrm{t}, 2 \mathrm{H}, 2 \times \mathrm{CH}_{2} \mathrm{OH}, J=5.19 \mathrm{~Hz}\right), 6.70-6.93(\mathrm{~m}, \\
6 \mathrm{H}, \mathrm{ArH}), 7.09-7.24(\mathrm{t}, 2 \mathrm{H}, \mathrm{ArH}, J=7.2 \mathrm{~Hz}), 10.33(\mathrm{~s}, 2 \mathrm{H}, 2 \times 1- \\
\mathrm{NH}) \text { ppm; EI-MS for } \mathrm{C}_{34} \mathrm{H}_{36} \mathrm{~N}_{6} \mathrm{O}_{8}(\mathrm{M}+1) 656.7 .\end{array}$ \\
\hline$\stackrel{\dot{\vec{I}}}{\dot{+}}$ & 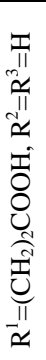 & 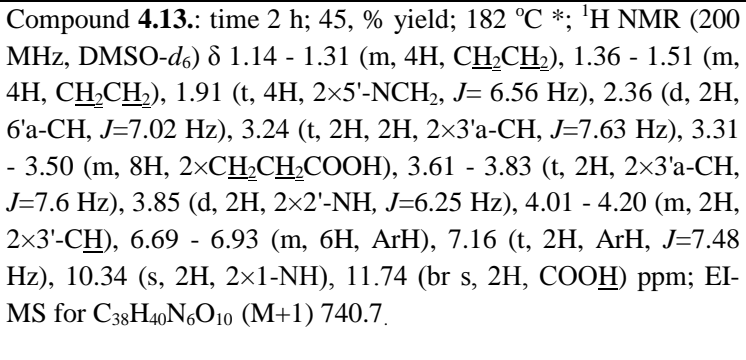 \\
\hline$\underset{\overrightarrow{+}}{\dot{+}}$ & 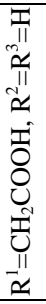 & 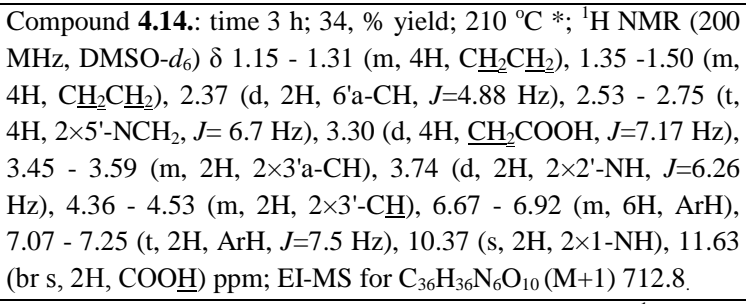 \\
\hline 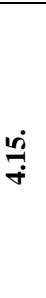 & 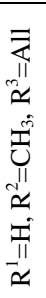 & $\begin{array}{l}\text { Compound 4.15.: time } 5 \mathrm{~h} ; 78, \% \text { yield; } 103-105{ }^{\circ} \mathrm{C} * ;{ }^{1} \mathrm{H} \mathrm{NMR} \\
\left(200 \mathrm{MHz}, \mathrm{DMSO}-d_{6}\right) \delta 1.12-1.27\left(\mathrm{~m}, 4 \mathrm{H}, \mathrm{C}_{2} \mathrm{C}_{2} \underline{\mathrm{H}}_{2}\right), 1.38- \\
1.47\left(\mathrm{~m}, 8 \mathrm{H}, \mathrm{C}_{2} \underline{\mathrm{H}}_{2} \mathrm{CH}_{2} 4 \mathrm{H}, 2 \times 5^{\prime}-\mathrm{NCH}_{2}\right), 1.88\left(\mathrm{~s}, 6 \mathrm{H}, 2 \times 2^{\prime}-\mathrm{NCH}_{3}\right), \\
3.23\left(\mathrm{~d}, 2 \mathrm{H}, 2 \times 6{ }^{\prime} \mathrm{a}-\mathrm{CH}, J=7.63 \mathrm{~Hz}\right), 3.48\left(\mathrm{t}, 2 \mathrm{H}, 2 \times 3{ }^{\prime} \mathrm{a}-\mathrm{CH} J=7.15\right. \\
\mathrm{Hz}), 4.27\left(\mathrm{~m}, 6 \mathrm{H}, 2 \times 3^{\prime}-\mathrm{CH}, 2 \times \underline{\mathrm{CH}}_{2} \mathrm{CH}=\mathrm{CH}_{2}\right), 5.12(\mathrm{~m}, 4 \mathrm{H}, \\
\left.2 \times \mathrm{CH}_{2} \mathrm{CH}=\mathrm{CH}_{2}\right), 5.78\left(\mathrm{~m}, 2 \mathrm{H}, 2 \times \mathrm{CH}_{2} \mathrm{CH}=\mathrm{CH}_{2}\right), 6.93(\mathrm{~m}, 6 \mathrm{H}, \\
\mathrm{ArH}), 7.28(\mathrm{t}, 2 \mathrm{H}, \mathrm{ArH}, J=7.5 \mathrm{~Hz}) \mathrm{ppm} \text {, EI-MS for } \mathrm{C}_{40} \mathrm{H}_{44} \mathrm{~N}_{6} \mathrm{O}_{6} \\
(\mathrm{M}+1) 704.8 .\end{array}$ \\
\hline
\end{tabular}




\section{Compound 4.16.: time $1.5 \mathrm{~h} ; 80$, \% yield; $208-210{ }^{\circ} \mathrm{C} ;{ }^{1} \mathrm{H}$ NMR

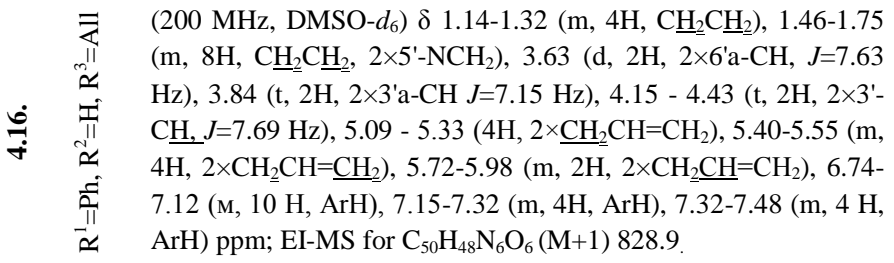
Compound 4.17.: time $40 \mathrm{~min} ; 47, \%$ yield; $120{ }^{\circ} \mathrm{C} * ;{ }^{1} \mathrm{H}$ NMR $\equiv \quad\left(200 \mathrm{MHz}, \mathrm{DMSO}-d_{6}\right) \delta 1.16-1.39\left(\mathrm{~m}, 4 \mathrm{H}, \mathrm{CH}_{2} \mathrm{CH}_{2}\right), 1.43-1.62$
$\quad\left(\mathrm{~m}, 4 \mathrm{H}, \mathrm{C}_{2} \mathrm{C}_{2} \underline{\mathrm{H}}_{2}\right), 1.79\left(8 \mathrm{H}, 2 \times 5{ }^{\prime}-\mathrm{NCH}_{2},\left(\mathrm{C}_{2}\right)_{3}\right), 2.11-2.28(\mathrm{~m}$, $\left.\approx 4 \mathrm{H},\left(\mathrm{C}_{2}\right)_{3}\right), 2.28-2.41\left(\mathrm{~m}, 4 \mathrm{H},\left(\underline{\mathrm{C}}_{2}\right)_{3}\right), 2.89(\mathrm{~d}, 2 \mathrm{H}, 2 \times 6 \mathrm{a}-\mathrm{C} \underline{\mathrm{H}}$, $\therefore \quad 7.17 \mathrm{~Hz}), 3.52\left(\mathrm{t}, 2 \mathrm{H}, 2 \times 3{ }^{\prime} \mathrm{a}-\mathrm{CH}, J=7.71 \mathrm{~Hz}\right), 4.07-4.37(\mathrm{~m}, 6 \mathrm{H}$,

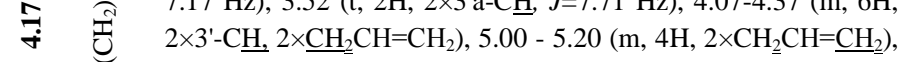 III $5.70-5.93\left(\mathrm{~m}, 2 \mathrm{H}, 2 \times \mathrm{CH}_{2}-\mathrm{CH}=\mathrm{CH}_{2}\right), 6.76-7.04(\mathrm{~m}, 6 \mathrm{H}, \mathrm{ArH})$, 7.14-7.38 (m, $2 \mathrm{H}, \mathrm{ArH})$ ppm; EI-MS for $\mathrm{C}_{44} \mathrm{H}_{48} \mathrm{~N}_{6} \mathrm{O}_{6}(\mathrm{M}+1)$ 756.9 .

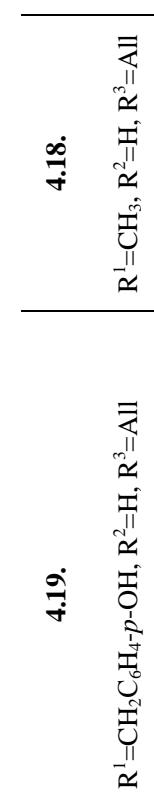

Compound 4.18.: time $3 \mathrm{~h} ; 51, \%$ yield; $150{ }^{\circ} \mathrm{C} * ;{ }^{1} \mathrm{H}$ NMR (200 $\mathrm{MHz}$, DMSO- $\left.d_{6}\right) \delta 1.16\left(\mathrm{~d}, 6 \mathrm{H}, 2 \times \mathrm{CH}_{3}, J=6.10 \mathrm{~Hz}\right), 1.14-1.33$ (m, $4 \mathrm{H}, \mathrm{CH}_{2} \mathrm{CH}_{2}$ ), 1.35-1.53 (m, 8H, $\underline{\mathrm{CH}}_{2} \mathrm{CH}_{2}, 2 \times 5^{\prime}-\mathrm{NCH}_{2}$ ), 3.17 - 3.28 (d, $2 \mathrm{H}, 2 \times 6$ 'a-CH, J=7.70 Hz), $3.60-3.83$ (m, 4H, $2 \times 3$ 'a$\left.\mathrm{CH}, 2 \times 2^{\prime}-\mathrm{NH}\right), 4.16-4.40\left(\mathrm{~m}, 6 \mathrm{H}, 2 \times 3^{\prime}-\mathrm{CH}, 2 \times \underline{\mathrm{CH}}_{2} \mathrm{CH}=\mathrm{CH}_{2}\right)$, 5.04-5.28 (m, 4H, $\left.2 \times \mathrm{CH}_{2} \mathrm{CH}=\underline{\mathrm{CH}}_{2}\right), \quad 5.67-5.96(\mathrm{~m}, 2 \mathrm{H}$, $\left.2 \times \mathrm{CH}_{2} \mathrm{CH}=\mathrm{CH}_{2}\right), 6.73-7.02(\mathrm{~m}, 6 \mathrm{H}, \mathrm{ArH}), 7.10-7.33(\mathrm{~d}, 2 \mathrm{H}$, ArH, $J=7.0 \mathrm{~Hz}$ ) ppm; EI-MS for $\mathrm{C}_{40} \mathrm{H}_{44} \mathrm{~N}_{6} \mathrm{O}_{6}(\mathrm{M}+1) 704.8$. Compound 4.19.: time $1.5 \mathrm{~h} ; 51, \%$ yield; $230{ }^{\circ} \mathrm{C} * ;{ }^{1} \mathrm{H}$ NMR $\left(200 \mathrm{MHz}, \mathrm{DMSO}-d_{6}\right) \delta 1.21-1.43\left(\mathrm{~m}, 4 \mathrm{H}, \mathrm{CH}_{2} \mathrm{C}_{2} \underline{H}_{2}\right), 1.45-1.68$ (m, $\left.4 \mathrm{H}, \mathrm{C}_{2} \mathrm{C}_{2} 22 \times 5^{\prime}-\mathrm{NCH}_{2}\right), 3.20-3.48$ (d, $2 \mathrm{H}, 2 \times 6$ 'a-CH, $J=7.63 \mathrm{~Hz}), 3.57(\mathrm{t}, 2 \mathrm{H}, 2 \times 3 \mathrm{a}-\mathrm{C} \underline{\mathrm{H}}, J=7.71 \mathrm{~Hz}), 3.67-3.85$ (d, $\left.2 \mathrm{H}, 2 \times 2^{\prime}-\mathrm{NH}, \quad J=6.02 \mathrm{~Hz}\right), \quad 4.10-4.25\left(\mathrm{~m}, 6 \mathrm{H}, 2 \times 3^{\prime}-\mathrm{C} \underline{\mathrm{H}}\right.$, $\left.2 \times \mathrm{CH}_{2} \mathrm{C}_{6} \mathrm{H}_{4}-4-\mathrm{OH}\right), 4.27-4.45\left(4 \mathrm{H}, 2 \times \underline{\mathrm{CH}}_{2} \mathrm{CH}=\mathrm{CH}_{2}\right), 5.06-$ $5.30 \quad\left(\mathrm{~m}, \quad 4 \mathrm{H}, \quad 2 \times \mathrm{CH}_{2} \mathrm{CH}=\mathrm{CH}_{2}\right), \quad 5.68-5.94 \quad(\mathrm{~m}, \quad 2 \mathrm{H}$, $\left.2 \times \mathrm{CH}_{2} \underline{\mathrm{CH}}=\mathrm{CH}_{2}\right), 6.61-6.65$ (d, 4H, ArH, J=7.94 Hz), 6.79 - 7.13 (m, 10H, ArH), 7.20 (d, 2H, ArH, J=7.02 Hz), 9.15 (br., s, 2H, $\left.2 \times \mathrm{CH}_{2} \mathrm{C}_{6} \mathrm{H}_{4}-4-\mathrm{OH}\right) \quad$ ppm; ${ }^{13} \mathrm{C}-\mathrm{NMR}\left(75 \mathrm{MHz}\right.$, DMSO- $\left.d_{6}\right) \delta$ $25.33,25.61,26.96,27.05,27.78,36.08,36.85,38.01,38.71$, $38.92,39.13,39.55,39.75,39.96,41.15,47.88,52.06,59.27$, $61.90,67.20,108.57,114.84,116.87,121.45,125.60,126.86$, $128.79,129.67,129.86,131.90,134.27,142.58,155.40,170.93$, 174.61, 176.29, 178.17 ppm; EI-MS for $\mathrm{C}_{52} \mathrm{H}_{52} \mathrm{~N}_{6} \mathrm{O}_{8}(\mathrm{M}+1)$ 889.0.

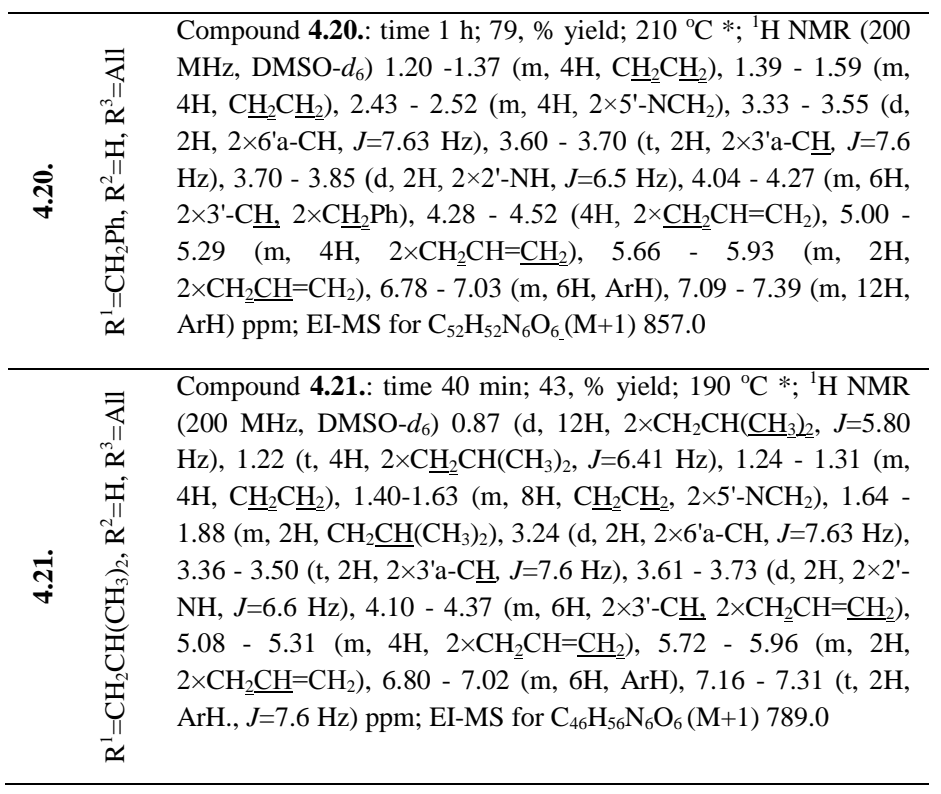

\begin{tabular}{|c|c|c|}
\hline ત્ & 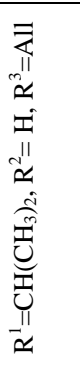 & 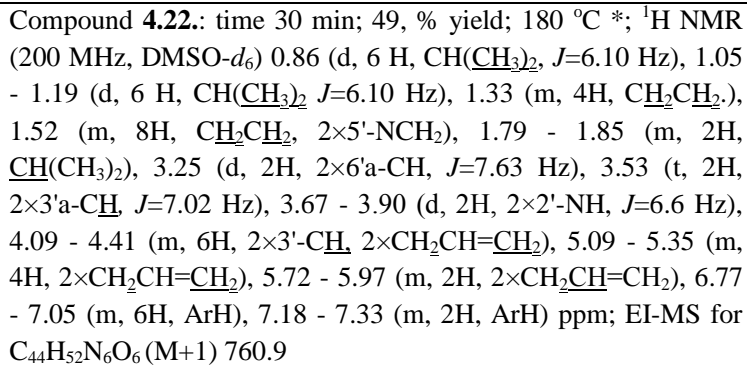 \\
\hline$\stackrel{\text { }}{\dot{\tau}}$ & 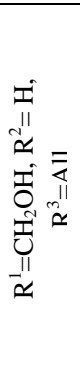 & 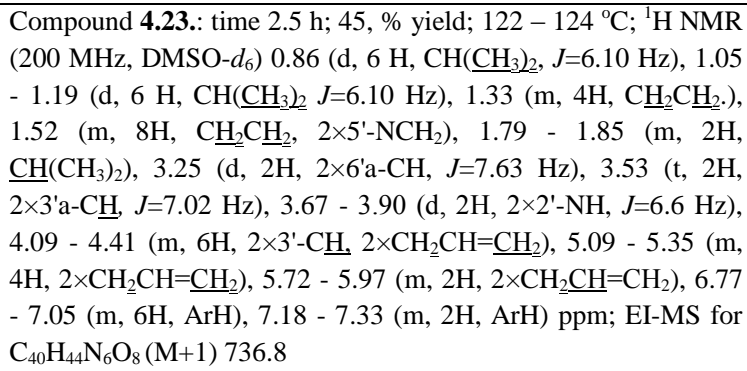 \\
\hline$\underset{\sim}{\stackrel{\text { I }}{+}}$ & 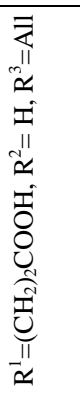 & 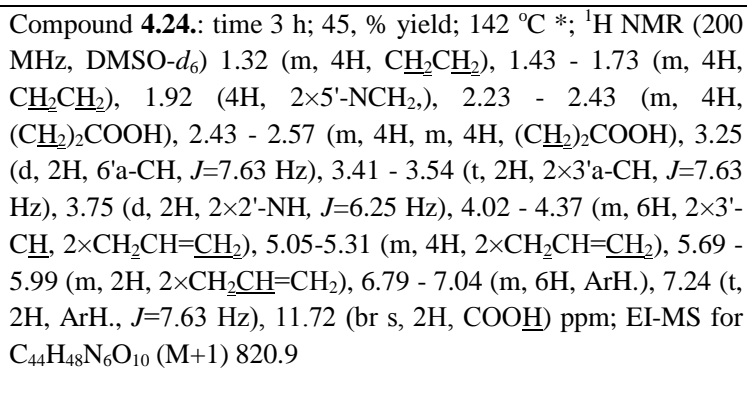 \\
\hline ஸ் & 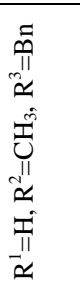 & $\begin{array}{l}\text { Compound 4.25.: time } 5 \mathrm{~h} ; 46, \% \text { yield; } 140{ }^{\circ} \mathrm{C} * ;{ }^{1} \mathrm{H} \text { NMR }(200 \\
\left.\mathrm{MHz}, \mathrm{DMSO}-d_{6}\right) 1.19-1.33\left(\mathrm{~m}, 4 \mathrm{H}, \mathrm{C}_{2} \mathrm{C}_{2} \underline{\mathrm{H}}_{2}\right), 1.46-1.55(\mathrm{~m}, \\
\left.4 \mathrm{H}, \mathrm{C}_{2} \mathrm{C}_{2} \underline{\mathrm{H}}_{2}\right), 1.89\left(\mathrm{~s}, 6 \mathrm{H}, 2 \times 2^{\prime}-\mathrm{NC}_{3}\right), 2.41-2.53\left(\mathrm{t}, 4 \mathrm{H}, 2 \times 5^{\prime}-\right. \\
\left.\mathrm{NCH}_{2}, J=7.1 \mathrm{~Hz}\right), 3.21-3.48\left(\mathrm{~d}, 2 \mathrm{H}, 2 \times 6{ }^{\prime} \mathrm{a}-\mathrm{CH}, J=7.6 \mathrm{~Hz}\right), 3.58 \\
(\mathrm{~m}, 2 \mathrm{H}, 2 \times 3 \text { 'a-CH, } J=6.10 \mathrm{~Hz}), 3.74-4.33\left(\mathrm{~d}, 4 \mathrm{H}, 2 \times 3^{\prime}-\mathrm{CH}_{2}\right), \\
4.79-4.99\left(\mathrm{~s}, 4 \mathrm{H}, 2 \times \mathrm{C}_{2} \mathrm{Ph}\right), 6.71-6.85(\mathrm{~m}, 5 \mathrm{H}, \mathrm{ArH}), 6.85- \\
7.03(\mathrm{~m}, 3 \mathrm{H}, \mathrm{ArH}), 7.15-7.43(\mathrm{~m}, 10 \mathrm{H}, \mathrm{ArH}) \mathrm{ppm} \text {; EI-MS for } \\
\mathrm{C}_{48} \mathrm{H}_{48} \mathrm{~N}_{6} \mathrm{O}_{6}(\mathrm{M}+1) 804.9\end{array}$ \\
\hline ஸें & 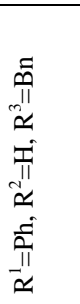 & 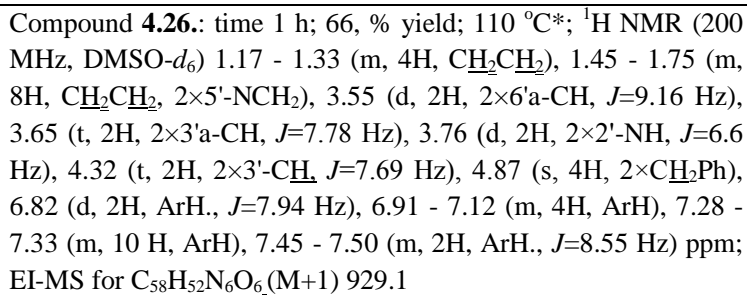 \\
\hline 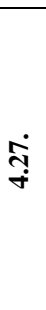 & 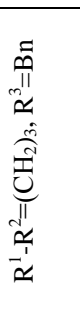 & $\begin{array}{l}\text { Compound 4.27.: time } 35 \mathrm{~min} ; 50, \% \text { yield; } 220{ }^{\circ} \mathrm{C}^{*} ;{ }^{1} \mathrm{H} \text { NMR } \\
\left(200 \mathrm{MHz}, \mathrm{DMSO}-d_{6}\right) 1.17-1.33\left(\mathrm{~m}, 4 \mathrm{H}, \mathrm{C}_{2} \mathrm{C}_{2}{ }_{2}\right), 1.45-1.75 \\
\left(\mathrm{~m}, 8 \mathrm{H}, \mathrm{C}_{2} \mathrm{CH}_{2}, 2 \times 5^{\prime}-\mathrm{NCH}_{2}\right), 3.55(\mathrm{~d}, 2 \mathrm{H}, 2 \times 6 \mathrm{a}-\mathrm{CH}, J=9.16 \\
\mathrm{Hz}), 3.65\left(\mathrm{t}, 2 \mathrm{H}, 2 \times 3^{\prime} \mathrm{a}-\mathrm{CH}, J=7.78 \mathrm{~Hz}\right), 3.76\left(\mathrm{~d}, 2 \mathrm{H}, 2 \times 2^{\prime}-\mathrm{NH},\right. \\
J=6.6 \mathrm{~Hz}), 4.32\left(\mathrm{t}, 2 \mathrm{H}, 2 \times 3^{\prime}-\mathrm{CH}, \underline{H}_{2}=7.69 \mathrm{~Hz}\right), 4.87(\mathrm{~s}, 4 \mathrm{H}, \\
\left.2 \times \mathrm{C}_{2} \mathrm{Ph}\right), 6.82(\mathrm{~d}, 2 \mathrm{H}, \mathrm{ArH} ., J=7.94 \mathrm{~Hz}), 6.91-7.12(\mathrm{~m}, 4 \mathrm{H}, \\
\mathrm{ArH}), 7.28-7.33(\mathrm{~m}, 10 \mathrm{H}, \mathrm{ArH}), 7.45-7.50(\mathrm{~m}, 2 \mathrm{H}, \mathrm{ArH} \text {, } \\
J=8.55 \mathrm{~Hz}) \text { ppm; EI-MS for } \mathrm{C}_{52} \mathrm{H}_{52} \mathrm{~N}_{6} \mathrm{O}_{6}(\mathrm{M}+1) 857.0\end{array}$ \\
\hline$\underset{\sim}{\stackrel{\leftrightarrow}{+}}$ & 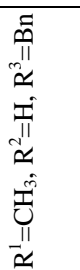 & $\begin{array}{l}\text { Compound 4.28.: time } 1 \mathrm{~h} ; 81, \% \text { yield; } 190{ }^{\circ} \mathrm{C}^{*} ;{ }^{1} \mathrm{H} \text { NMR }(200 \\
\left.\mathrm{MHz}, \mathrm{DMSO}-d_{6}\right) 1.18\left(\mathrm{~d}, 6 \mathrm{H}, 2 \times \mathrm{CH}_{3}, J=6.41 \mathrm{~Hz}\right), 1.28-1.32 \\
\left(\mathrm{~m}, 4 \mathrm{H}, \mathrm{CH}_{2} \mathrm{CH}_{2}\right), 1.46-1.53\left(\mathrm{~m}, 4 \mathrm{H}, \mathrm{CH}_{2} \mathrm{C}_{2}\right), 3.35-3.49(\mathrm{~m}, \\
\left.6 \mathrm{H}, 2 \times 3{ }^{\prime} \mathrm{a}-\mathrm{CH}, 2 \times 5^{\prime}-\mathrm{NCH}_{2}\right), 3.69-3.89\left(\mathrm{~d}, 2 \mathrm{H}, 2 \times 2^{\prime}-\mathrm{NH}, J=4.88\right. \\
\mathrm{Hz}), 4.35\left(\mathrm{~m}, 2 \mathrm{H}, 2 \times 3{ }^{\prime}-\mathrm{CH}\right), 4.83\left(\mathrm{~s}, 4 \mathrm{H}, 2 \times \mathrm{CH}_{2} \mathrm{Ph}\right), 6.75-7.00 \\
(\mathrm{~m}, 4 \mathrm{H}, \mathrm{ArH}), 7.09-7.40(\mathrm{~m}, 14 \mathrm{H}, \mathrm{ArH}) \mathrm{ppm} \text { EI-MS for } \\
\mathrm{C}_{48} \mathrm{H}_{48} \mathrm{~N}_{6} \mathrm{O}_{6}(\mathrm{M}+1) 804.9\end{array}$ \\
\hline
\end{tabular}




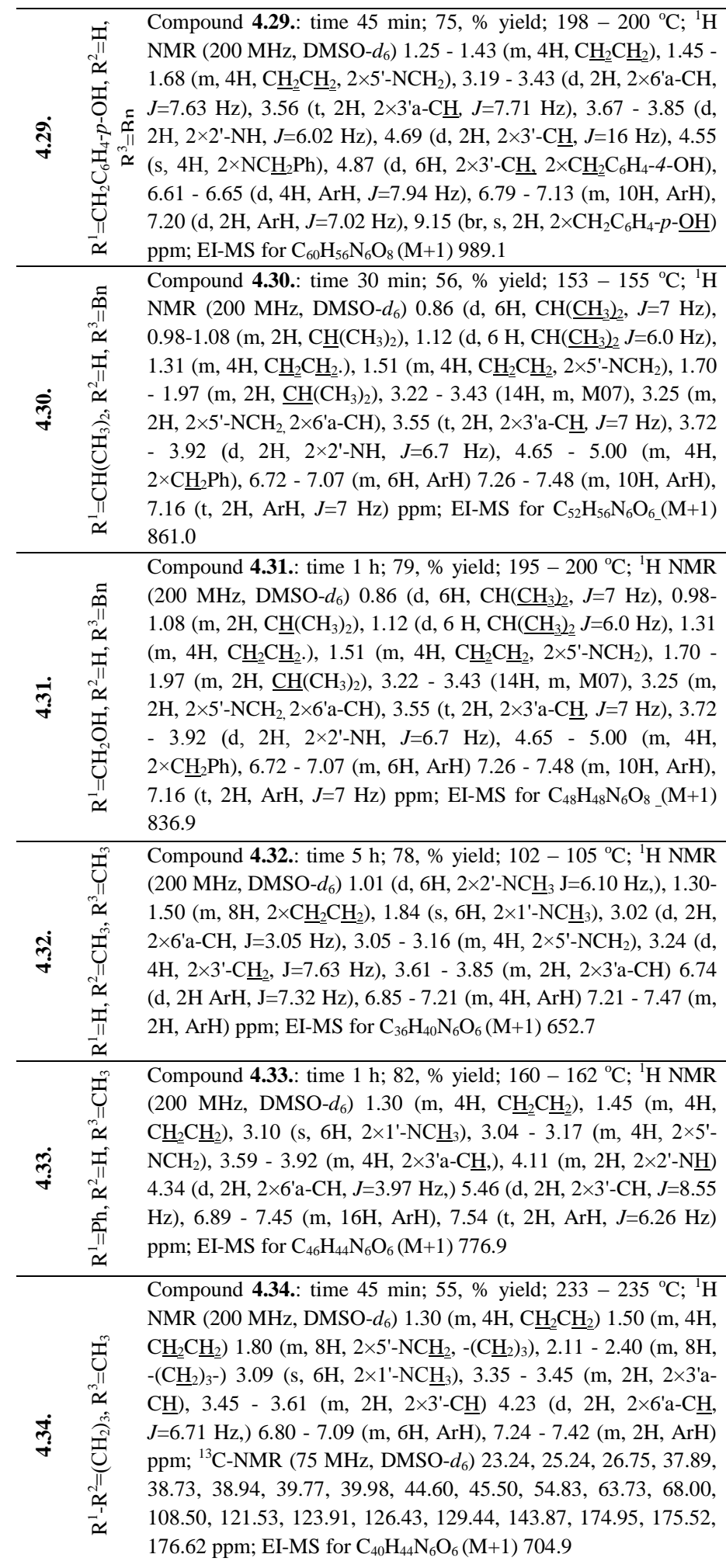

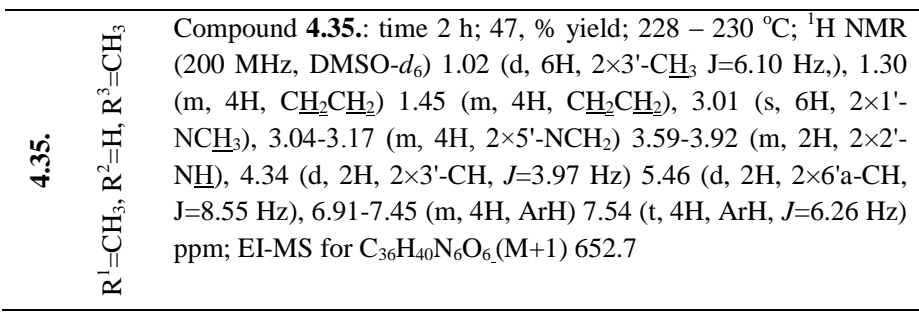

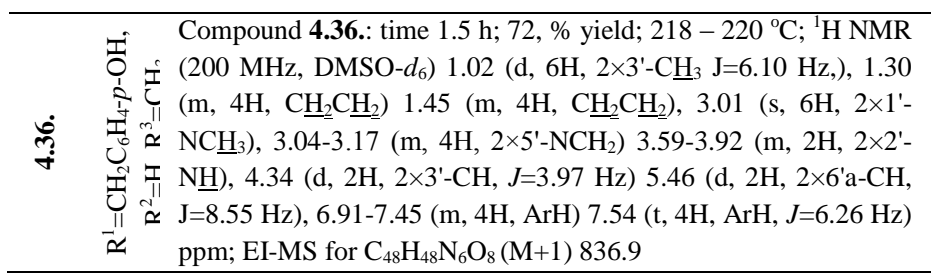

In the course of the studies, we examined antibacterial properties of 36 synthesized substances including some novel spirooxindole derivatives $\mathbf{4 . 1 - 4 . 3 6}$ against different types of microorganisms (gram-positive and gram-negative). Most of the tested compounds showed a weak antimicrobial activity, and the most effective growth inhibitors are presented in Table 3

Seven reference strains of microorganisms were used as control ones: Escherichia coli ATCC 25922, Enterobacter cloacae №487, Klebsiella pneumoniae №247, Pseudomonas aeruginosa ATCC 27853, Staphylococcus aureus ATCC 25923, Staphylococcus epidermidis No.235, Candida albicans ATCC653/885. The microorganisms belong to different groups: gram-negative bacteria from the Enterobacteriaceae family ( $E$. coli, E. cloacae, K. pneumoniae) while P. aeruginosa belong to non-fermenting gram-negative bacteria. Gram-positive cocci $S$. aureus and $S$. epidermidis belong to the Micrococcaceae family, and fungi $C$. albicans to the Criptococcaceae family. In order to detect antimicrobial properties of the compounds, the value of the minimal inhibitory concentration (MIC) of aqueous solutions of these 4.1-4.36 substances in five double dilutions $100 \mu \mathrm{g} / \mathrm{mL}, 50$ $\mu \mathrm{g} / \mathrm{mL}, 25 \mu \mathrm{g} / \mathrm{mL}, 12.5 \mu \mathrm{g} / \mathrm{mL}, 6.25 \mu \mathrm{g} / \mathrm{mL}, 3.125 \mu \mathrm{g} / \mathrm{mL}$ was determined.

Compounds 4.4, 4.5, 4.11, 4.15, 4.19, 4.21, 4.24, 4.25, 4.31 demonstrated the maximum activity against gram-positive microorganisms and are not active against gram-negative bacteria. Only compound $4.12\left(\mathrm{R}^{1}=\mathrm{CH}_{2} \mathrm{OH}, \mathrm{R}^{2}=\mathrm{R}^{3}=\mathrm{H}\right)$ demonstrated a relatively weak growth inhibition of $C$. albicans. The $N^{1}$ benzyleted derevated substance $4.31\left(\mathrm{R}^{1}=\mathrm{CH}_{2} \mathrm{OH}, \mathrm{R}^{2}=\mathrm{H}, \mathrm{R}^{3}=\mathrm{Bn}\right)$ was found to be the most potent compound with antibacterial activity against $S$. aureus and $S$. epidermidis and $C$. albicans with MIC $6.25 \mu \mathrm{g} / \mathrm{mL}$.

Compound $4.4\left(\mathrm{R}^{1}=\mathrm{H}, \mathrm{R}^{2}=\mathrm{CH}_{3}, \mathrm{R}^{3}=\mathrm{H}\right)$ showed activity only with respect to staphylococci. Although none of the tested compounds was surpassed by its active reference-drugs comparison (Ciproflaxacin and Fluconazole), the obtained results make it possible to construct more active antimicrobial compounds based on bis-spirooxindoles structures in the future. It was found that test-strains of the Micrococcaceae family were more susceptible to the test compounds.

The important physicochemical properties (lipophilicity $(\log \mathrm{P})$, topological molecular polar surface area (TPSA) and molar volume) for all most active compounds $4.4,4.5,4.11,4.15$, 4.19, 4.21, 4.24, 4.25, 4.31 were calculated by the Molinspiration server (Molinspiration property engine v2016.10) for the establishing structure activity relationship (SAR) and listed in Table 4 
Table 3: Antimicrobial screening data for the most active compounds and MIC $(\mu \mathrm{g} / \mathrm{ml})$.

\begin{tabular}{|c|c|c|c|c|c|c|c|}
\hline \multirow{3}{*}{ Compound. № } & \multicolumn{7}{|c|}{$M I C^{I}, \mu \mathrm{g} / \mathrm{mL}$} \\
\hline & \multicolumn{2}{|c|}{ Gram "+ve" bacteria } & \multicolumn{4}{|c|}{ Gram "-ve" bacteria } & \multirow{2}{*}{$\begin{array}{c}\text { Fungal strain } \\
\text { C. albicans }\end{array}$} \\
\hline & S. aureus & S. epidermidis & E. coli & P. aeruginosa & K. pneumoniae & E. cloacae & \\
\hline 4.4. & 6.25 & 6.25 & growth $^{2}$ & growth & growth & growth & growth \\
\hline 4.31. & 6.25 & 6.25 & growth & growth & growth & growth & 6.25 \\
\hline 4.25. & 50 & 50 & growth & growth & growth & growth & growth \\
\hline 4.19. & 50 & 25 & growth & growth & growth & growth & 100 \\
\hline 4.5. & 100 & 100 & growth & growth & growth & growth & 50 \\
\hline 4.11. & 100 & 50 & growth & growth & growth & growth & growth \\
\hline 4.24. & 100 & 100 & growth & growth & growth & growth & growth \\
\hline 4.15. & 100 & 100 & growth & growth & growth & growth & 100 \\
\hline 4.21. & 100 & 25 & growth & growth & growth & growth & growth \\
\hline 4.12. & 100 & 100 & growth & growth & growth & growth & 100 \\
\hline Control & growth & growth & growth & growth & growth & growth & growth \\
\hline Ciproflox. ${ }^{4}$ & 0.78 & 2 & 0.39 & 1.0 & 0.78 & 2 & $\mathrm{~N} / \mathrm{T}$ \\
\hline Fluconazole ${ }^{5}$ & $\mathrm{~N} / \mathrm{T}^{3}$ & $\mathrm{~N} / \mathrm{T}$ & $\mathrm{N} / \mathrm{T}$ & $\mathrm{N} / \mathrm{T}$ & $\mathrm{N} / \mathrm{T}$ & $\mathrm{N} / \mathrm{T}$ & 3.125 \\
\hline
\end{tabular}

Notes:

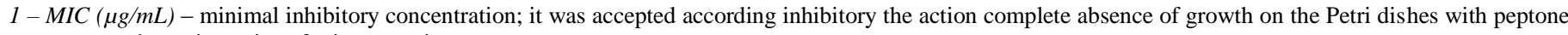
meat agar and certain strains of microorganisms;

2 - "growth" - it is that investigated compound was not active in concentrations $100 \mu \mathrm{g} / \mathrm{ml}$;

Control - DMSO solution in peptone meat agar $(100 \mu \mathrm{g} / \mathrm{ml})$;

$3-\mathrm{N} / \mathrm{T}-$ it was not tested.

4 - Ciproflox. - Ciproflaxacin DMSO solution;

5 - Fluconazole water solution

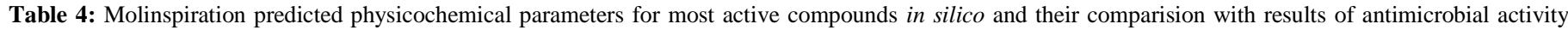
investigation in vitro.

\begin{tabular}{|c|c|c|c|c|c|c|}
\hline \multirow{2}{*}{ Compound. } & \multirow{2}{*}{$\log P$} & \multirow{2}{*}{$\begin{array}{c}\text { TPSA, } \\
\AA^{2} \\
\end{array}$} & \multirow{2}{*}{$\begin{array}{c}\text { Molecular volume } \\
(\mathrm{MV}), \AA^{3}\end{array}$} & \multicolumn{3}{|c|}{$M I C, \mu \mathrm{g} / \mathrm{mL}$ (in vitro) } \\
\hline & & & & S. aureus & S. epidermidis & C. albicans \\
\hline 4.4 . & 1.65 & 157.01 & 547.39 & 6.25 & 6.25 & growth \\
\hline 4.31 . & 2.99 & 179.89 & 741.09 & 6.25 & 6.25 & 6.25 \\
\hline 4.25 . & 4.94 & 139.43 & 724.57 & 50 & 50 & growth \\
\hline 4.19 . & 5.40 & 179.89 & 796.55 & 50 & 25 & 100 \\
\hline 4.5. & 3.62 & 197.47 & 706.72 & 100 & 100 & 50 \\
\hline 4.11 . & 3.21 & 157.01 & 614.16 & 100 & 50 & growth \\
\hline 4.24. & 2.32 & 214.03 & 725.30 & 100 & 100 & growth \\
\hline 4.15 . & 3.26 & 121.85 & 637.93 & 100 & 100 & 100 \\
\hline 4.21 & 6.05 & 139.43 & 737.60 & 100 & 25 & growth \\
\hline 4.12 . & -0.30 & 197.47 & 563.90 & 100 & 100 & 100 \\
\hline
\end{tabular}

From the obtained results, we tentatively assume that the activity may relate to contribution of the lipophilicity $(\operatorname{LogP})$, topological molecular polar surface area (TPSA) and molecular volume (MV) descriptors. Molinspiration server has predicted that $\mathrm{N}$-benzyleted derevated substance $\mathbf{4 . 3 1}$ is moderately lipophilic $(\operatorname{LogP}=2.99)$ while 4.12 is hydrophylic $(\log P=-0.30)$ and it has more polarity (TPSA $=197.47 \AA^{2}$ ).

Obviously, more polar compound $\mathbf{4 . 1 2}$ exhibit less lipid solubility that limits cell membrane penetration (Waterhouse, 2003). Since all the compounds studied have the same bisspirooxindolic system, the changes in the molar volume depend only on the structure of the substituents $\mathrm{R}^{1}, \mathrm{R}^{2}, \mathrm{R}^{3}$.

This indicates that the antimicrobial activity of those compounds depends not only on the length of the longer substituents, but also on the presence of the second one that contributes to the hydrophobicity, polar surface area (TPSA) and on molecular volume of the whole molecule.
Therefore, a comparison of structures 4.12 (MV=563.90 $\left.\AA^{3}\right)$ and 4.31 (MV=741.09 $\left.\AA^{3}\right)$ shows that the introduction of a benzyl radical dramatically increases the molecular volume and lipophilicity. Nonetheless, that reduces the polarity of the molecule as a whole and it is possible to form chelate complexes with microelements $(\mathrm{Mg}, \mathrm{Fe}, \mathrm{Cu})$ and DNA of microbes resulting in enzyme systems inhibition (Fig. 3). However, more analogues have to be compared to establish a proper structure-activity relationship.

The combination of new substituents should lead to an optimal balance of lipophilicity, polarity, and molecular volume. Further extensive studies are necessary to determine additional physicochemical and biological parameters, get deeper insight the structure-activity relationship and optimize the effectiveness and anti-staphylococci activity of hexamethylene-N,N'-bis-derivatives of $3 \mathrm{a}^{\prime}, 6 \mathrm{a}^{\prime}$-dihydro-2' $H$-spiro[indole-3,1'-pyrrolo[3,4-c]pyrrole]$2,4^{\prime}, 6^{\prime}\left(1 H, 3^{\prime} H, 5^{\prime} H\right)$-trione series of molecules. 


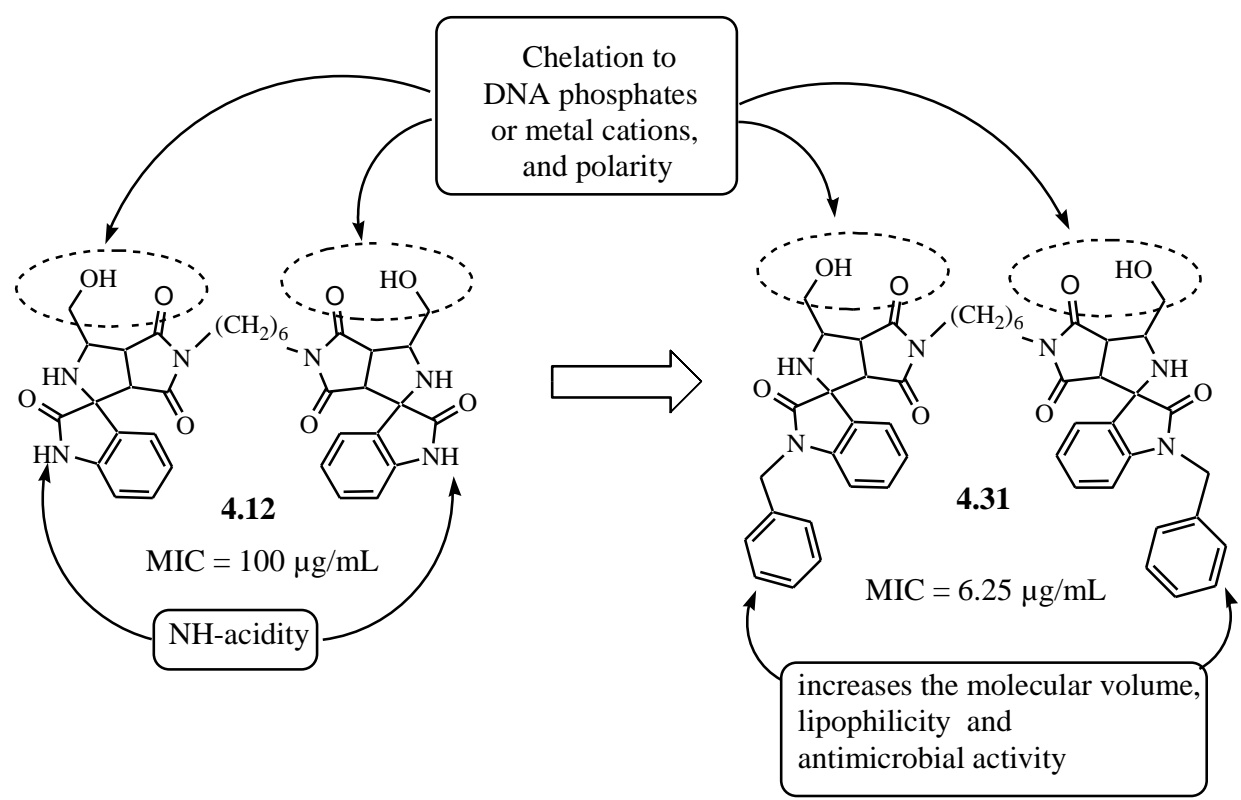

Fig. 3: Structure Activity Relationship illustration.

\section{CONCLUSION}

In summary, we have successfully developed a 1,3dipolar cycloaddition of 1,6-bismaleimidohexane to azomethine ylides generated in situ from isatins and some $\alpha$-amino acids, and 36 novel hexamethylene-N,N'-bis-derivatives of $3 \mathrm{a}^{\prime}, 6 \mathrm{a}^{\prime}$-dihydro2' $H$-spiro[indole-3,1'-pyrrolo[3,4-c]pyrrole]-2,4',6' $\left(1 H, 3^{\prime} H, 5^{\prime} H\right)$ trione 4.1-4.36 were synthesized and their structures were established by NMR, Mass and elemental analysis. The isolated yields in this one-pot protocol were moderate to high (43-88\%) and provided the desired target compounds with a high purity after a simple filtration and recrystallization from an ethanol/DMF mixture (1:1). This method has the advantages of convenient operation, mild reaction conditions and high efficiency. It has the advantages of mild reaction conditions, high atom economy and excellent yields. The most suitable conditions for this reaction were boiling in the alcoholic-water media. It was interesting to notice that test compound 4.31 with $\mathrm{N}^{1}$-benzyl and $\mathrm{R}^{1}=\mathrm{CH}_{2} \mathrm{OH}$ radicals led to a significant increase in antibacterial activity. Some obtained compounds showed a weak antimicrobial activity with regard to the Micrococcaceae family.

\section{ACKNOWLEDGEMENTS}

The author gratefully acknowledges Dr. Elena Poddubna and Prof. Vasiliy Zhadinskiy, Head of the Microbiology Department, Donetsk National Medical University (Ukraine), for providing microbiological experiment to carry out this study.

Financial support and sponsorship: The authors acknowledge National University of Pharmacy for financial support within the research program "Organic synthesis and analysis of bioactive compounds to develop drugs based on synthetic and semisynthetic substances" (Number of Ukraine state registration of research: 0114U000943).

Conflict of Interests: The authors confirm that this article content has no conflict of interest.

\section{REFERENCES}

Aliasghar J, Dariush K, Clercq D, Chanaz S. Synthesis, antibacterial, antifungal and antiviral activity, Evaluation of some new bisSchiff bases of isatin and their derivatives. Molecules, 2007; 12:17201730

Arun $\mathrm{Y}$, Bhaskar $\mathrm{G}$, Balachandran $\mathrm{C}$, Ignacimuthu $\mathrm{S}$, Perumal PT. Facile one-pot synthesis of novel dispirooxindole-pyrrolidine derivatives and their antimicrobial and anticancer activity against A549 human lung adenocarcinoma cancer cell line. Bioorganic \& medicinal chemistry letters, 2013; 23 (6):1839-1845.

Babu ARS, Raghunathan, R, Mathivanan N, Omprabha G, Velmurugan D, Raghu R. Synthesis, characterisation, anti-microbial activity and docking studies of novel Dispiro-Oxindolopyrrolidines, Current Chemical Biology, 2008; 2: 312-320.

Ball-Jones NR, Badillo JJ, Franz AK. Strategies for the enantioselective synthesis of spirooxindoles. Org. Biomol. Chem., 2012, 10, 5165-5181

Balouiri M, Sadiki M, Ibnsouda SK. Methods for in vitro evaluating antimicrobial activity: A review. J Pharm Analysis, 2016; 6(2): 71-79.

Harikrishna S, Ravindranath LK. Synthesis, characterization and antimicrobial activities of $\mathrm{N}$-substituted indoline derivatives of sultams. Der Pharma Chemica, 2015; 7 (1): 62-67.

Joaquim DS, Garden SJ, Pinto AC. The Chemistry of isatins: A Review. J. Braz. Chem. Soc, 2001; 12:273-324.

Karki SS, Hazare R, Kumar S, Saxena A, Katiyar A. Synthesis and antimicrobial activity of some 3-substituted-2-oxindole derivatives. Turk J Pharm Sci, 2011; 8 (2):169-178.

Osolodkin DI, Palyulin VA, Zefirov NS. Glycogen synthase kinase 3 as an anticancer drug target: novel experimental findings and trends in the design of inhibitors. Curr Pharm Des, 2013; 19(4):665-79. 
Pandeya SN, Gnana S, Saravanan M, Sriram D, Senthil K. Synthesis and antibacterial activity of Mannich bases of ciprofloxacin and lomefloxacin with isatin and its derivatives. Indian J Pharm. Sci, 1998; 60:280-282.

Pandeya SN, Smitha S, Jyoti M, Sridhar SK. Biological activities of isatin and its derivatives. Acta Pharm, 2005; 55:27-46.

Pandeya SN, Sriram D, Nath G, DeClercq E. Synthesis, antibacterial, antifungal and anti-HIV activities of Schiff and Mannich bases derived from isatin derivatives and $\mathrm{N}$-[4-(4'-chlorophenyl)thiazol-2yl] thiosemicarbazide. Eur J Pharm Sci, 1999; 9(1):25-31.

Patel JB, Cockerill FR, Bradford PA, Eliopoulos GM, Hindler JA, Jenkins SG, Lewis JS, Miller LA, Nicolau DP, Powell M, Swenson JM, Traczewski MM, Turnidge JD, Weinstein MP, Zimmer BL. 2015. Clinical and Laboratory Standards Institute. Performance Standards for Antimicrobial Susceptibility Testing; Twenty-Second Informational Supplement. Document M100-S25, Vol. 35, №3, CLSI, Wayne, PA, January, 2015 [ONLINE] Available at: http://shop.clsi.org/site/Sample_pdf/M100S25_sample.pdf [Accessed 08 January 2015].

Pavlovska TL, Redkin RGr, Lipson VV, Atamanuk DV. Molecular diversity of spirooxindoles. Synthesis and biological activity. Mol Divers, 2016; 20 (1):299-344.

Pavlovskaya TL, Redkin RGr, Yaremenko FG, Shishkina SV, Shishkin OV, Musatov VI, Lipson VV. Synthesis and Chemical Properties of New Derivatives of 3a',6a'-Dihydro-2'H-spiro[indole-3,1'-pyrrolo[3,4-

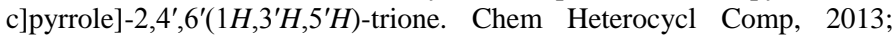
49:882-896

Pirrung C, Sunil V, Koushik D., Kathy A., Combinatorial optimization of isatin beta thiosemicarbazones as antipox virus agents. J. Med. Chem, 2005; 48:3045-3050.
Rane RA, Karunanidhi S, Jain K, Shaikh M, Hampannavar G, Karpoormath R. A Recent Perspective on Discovery and Development of Diverse Therapeutic Agents Inspired from Isatin Alkaloids. Curr Top Med Chem, 2016; 16(11):1262-89.

Singh GS, Desta ZY. Isatins as Privileged Molecules in Design and Synthesis of Spiro-Fused Cyclic Frameworks. Chem. Rev, 2012; 112 (11): 6104-6155.

Sumka EI, Redkin RG, Shemchuk LA, Chernykh VP, Yarmoluk SM. Screening and molecular properties of bis-derivatives of spiro[indole3,1 '-pyrrolo[3,4-c]pyrrole] in a search for potential inhibitors of protein kinases. Ukr Biopharm J, 2015; 6 (41):79-86.

Yu B, Yu DQ, Liu HM. Spirooxindoles: Promising scaffolds for anticancer agents. Eur J Med Chem, 2015; 5(97):673-98.

Waterhouse RN. Determination of lipophilicity and its use as a predictor of blood-brain barrier penetration of molecular imaging agents. Mol Imaging Biol, 2003; 5:376-389

\section{How to cite this article:}

Redkin RG, Syumka EI, Shemchuk LA, Chernykh VP. Synthesis And Antimicrobial Activity of Bis-Derivatives of 3a',6a'-Dihydro2'H-Spiro[Indole-3,1'Pyrrolo[3,4-c]Pyrrole]-2,4',6'(1H, 3'H, 5'H)Trione. J App Pharm Sci, 2017; 7 (06): 069-078. 